\title{
Educação em saúde como forma de prevenção do risco de queda nos idosos hospitalizados: um relato de experiência
}

\author{
Health education as a form to prevent the risk of falling in the hospitalized elderly: an \\ account of experience
}
Educación en salud como forma de prevención del riesgo de caída en los ancianos hospitalizados: un relato de experiencia

Bruna Fonseca Rezende ${ }^{1 *}$, Larissa Renata Bittencourt Pantoja ${ }^{1}$, Alícia Laura Lobo Modesto ${ }^{1}$, Beatriz Duarte de Oliveira ${ }^{1}$, Ruhan da Conceição Sacramento ${ }^{1}$, Maria da Conceição Nascimento Freitas ${ }^{1}$, Marcia Cristina Souza da Cruz ${ }^{1}$, Stephany Siqueira Braga ${ }^{1}$, Hennã Cardoso de Lima ${ }^{2}$, Everton Luís Freitas Wanzeler².

\section{RESUMO}

Objetivo: Sensibilizar os profissionais de saúde de uma clínica neurológica quanto à importância da avaliação do risco de queda no ambiente hospitalar por intermédio de uma ação de educação permanente. Relato da experiência: A pesquisa se deu a partir da observação sistemática da realidade em uma clínica de neurologia, sob a metodologia da problematização com o Arco de Maguerez. Durante a observação da realidade verificouse que a clínica não possuía nenhum método de avaliação quanto ao risco de queda, principalmente em pacientes idosos, sendo este o ponto-chave selecionado. Na etapa de teorização foi selecionado todo o arcabouço teórico relativo à temática de risco de queda nos idosos. Como hipótese de solução foi organizada uma ação de educação permanente acerca do risco de queda em idosos tendo como público alvo a equipe de enfermagem da clínica, sendo este o retorno à realidade. Considerações finais: $O$ presente estudo apresenta limitações, visto que a ação de educação permanente não foi realizada devido a questões do cotidiano da equipe do local. No entanto, a realização de ações educativas é imprescindível para a capacitação da equipe de enfermagem a fim de que os riscos sejam reduzidos, possibilitando a assistência segura ao paciente da $3^{\underline{a}}$ idade.

Palavras-chave: Enfermagem, Segurança do paciente, Saúde do idoso.

\section{ABSTRACT}

Objective: To sensitize the health professionals of a neurological clinic regarding the importance of evaluating the risk of falling in the hospital environment through a permanent education action. Experience report: The research was based on the systematic observation of reality in a neurology clinic, under the methodology of problematization with the Arch of Maguerez. During the observation of the reality it was verified that the clinic did not have any method of evaluation regarding the risk of falling, especially in elderly patients, being this the key point selected. In the theorizing stage, the whole theoretical framework regarding the risk of falling in the elderly subjects was selected. As a solution hypothesis, a permanent education action was organized about the risk of falls in the elderly, with the nursing team of the clinic as a target audience, which is the return to reality. Final considerations: The present study presents limitations since the action of permanent education was not carried out due to daily issues of the local team. However, the implementation of educational actions is essential for the training of the nursing team so that risks are reduced, enabling the safe care of the elderly patient.

Key words: Nursing, Patient safety, Health of the elderly.

1 Universidade do Estado do Pará (UEPA), Belém - PA. *E-mail: enfermagemsub01@gmail.com

²Universidade da Amazônia (UNAMA), Belém - PA. 


\section{RESUMEN}

Objetivo: Sensibilizar a los profesionales de salud de una clínica neurológica en cuanto a la importancia de la evaluación del riesgo de caída en el ambiente hospitalario por intermedio de una acción de educación permanente. Relato de experiencia: La investigación se dio a partir de la observación sistemática de la realidad en una clínica de neurología, bajo la metodología de la problematización con el Arco de Maguerez. Durante la observación de la realidad se verificó que la clínica no poseía ningún método de evaluación en cuanto al riesgo de caída, principalmente en pacientes ancianos, siendo éste el punto clave seleccionado. En la etapa de teorización fue seleccionado todo el marco teórico relativo a la temática de riesgo de caída en los ancianos. Como hipótesis de solución se organizó una acción de educación permanente acerca del riesgo de caída en ancianos teniendo como público objetivo el equipo de enfermería de la clínica, siendo éste el retorno a la realidad. Consideraciones finales: El presente estudio presenta limitaciones, ya que la acción de educación permanente no fue realizada debido a cuestiones del cotidiano del equipo del local. Sin embargo, la realización de acciones educativas es imprescindible para la capacitación del equipo de enfermería a fin de que los riesgos sean reducidos, posibilitando la asistencia segura al paciente de la tercera edad.

Palabras clave: Enfermería, Seguridad del paciente, Salud del anciano.

\section{INTRODUÇÃO}

No ambiente hospitalar, a queda é um evento frequente que pode comprometer a saúde e bem-estar físico e/ou mental do usuário em um serviço de saúde, e prejudicar a imagem do hospital que não consegue minimizar ou evitar esse evento adverso. Evento adverso (EA) é configurado como injúrias não intencionais decorrentes da atenção à saúde, não relacionadas à evolução natural da doença de base, que ocasionam lesões mensuráveis nos pacientes afetados. Diante do contexto hospitalar, as quedas são consideradas os principais eventos adversos, sendo responsáveis por $70 \%$ dos casos, considerando um índice que varia de 1,4 a 10,7 para cada 1000 pacientes por dia. Em clínicas de internação como a cirúrgica a queda é o segundo evento adverso mais observado (VICTOR MAG, et al., 2017; OLIVEIRA RM, et al., 2017).

Contudo, diversos instrumentos foram criados para a prevenção de quedas como a Escala de Quedas de Morse, desenvolvida em 1985 no Canadá, por Janice M. Morse, com base em um estudo perspectivo, com o objetivo de identificar e predizer as pessoas com risco de quedas fisiológicas. O acidente mais notificado a nível hospitalar e nos cuidados continuados é a queda do doente. Apesar dos avanços no processo de compreensão das quedas, estas continuam a ser um problema importante. A avaliação do risco de queda é intervenção essencial para a prevenção de quedas, sendo para isso importante a correta utilização da Escala de Quedas de Morse (EQM) (GARCIA MB, 2016).

A queda é configurada como o deslocamento não intencional do corpo de uma posição para outra de menor altura, podendo ou não o sujeito chegar ao chão, ou pode ser definida como a necessidade de apoio do sujeito ao deambular e/ou realizar atividades. A queda pode ocorrer da própria altura ou da cama, da cadeira, do assento sanitário, cadeira de rodas, entre outros. Alguns fatores predispõem ao maior risco para quedas, tais como confusão mental, desorientação, depressão, deficiência cognitiva, convulsão, desequilíbrio, astenia, deficiência motoras, uso de medicações que atuam em nível de sistema nervoso central, comprometimento sensorial (visão, audição ou tato) obesidade, história pregressa de queda e extremos de idade - idosos ( $>60$ anos) e crianças ( $<5$ anos) (BRASIL, 2013a).

A população idosa configura-se como um grupo de risco para queda devido às doenças prevalentes da idade, geralmente relacionadas ao sistema nervoso, doenças crônico-degenerativas, osteoporose e utilização de múltiplos medicamentos. Este grupo, muitas vezes, necessita de amparo hospitalar no que diz respeito a atenção à saúde, uma vez que segundo estudo realizado, pacientes acima de 65 anos ocupam mais de dois terços dos leitos hospitalares (SANTOS MGS, et al., 2010). A hospitalização aumenta o risco de queda, visto que os pacientes se encontram inseridos em ambientes que não são familiares e, muitas vezes, detém doenças que predispõem à queda ou necessitam de sedação, analgesias para alívio da dor ou qualquer outro medicamento, fazendo com que se tornem mais vulneráveis às quedas (SANTOS MGS, et al., 2010; PEREIRA ES, 2018; BRASIL, 2013a). 
Os danos provenientes de queda são inúmeros, dentre eles podemos destacar fraturas, hematomas subdurais, sangramentos e óbito. Além disso, pode também afetar o psicológico do cliente, visto que muitos apresentam insegurança e medo de novos episódios ocorrerem, se sentindo presos em suas próprias fragilidades. Entre outras consequências estão o aumento do tempo de internação gerando a elevação do custo de tratamento, causar descrédito com o cuidado prestado pela equipe de enfermagem, além de gerar implicações éticas e legais para as instituições (BRASIL, 2013a; VICTOR MAG, et al., 2017).

É imprescindível que os profissionais da saúde identifiquem os fatores de risco para quedas a fim de evitar agravos e a ocorrência da queda, assim como tratar os fatores etiológicos e comorbidades que possam surgir nesse processo. Impedir a ocorrência da queda atuando com a prevenção do risco é considerado hoje uma conduta de boa prática na assistência, tanto em hospitais quanto em instituições de longa permanência, sendo este um dos indicadores de qualidade (BRASIL, 2013a).

A segurança do paciente tornou-se um assunto de notória relevância para o sistema de saúde desde a década de 90, ocasionando um movimento global para a segurança do paciente. Em 2004, a Organização Mundial de Saúde (OMS) desenvolveu o projeto Aliança Mundial para a Segurança do Paciente, a fim de prevenir danos aos pacientes.

Em 2013, no Brasil, houve a criação do Programa Nacional de Segurança do Paciente (PNSP) que estabelece protocolos para a efetivação das metas internacionais de segurança, instituindo a formação de Núcleos de Segurança do Paciente dentro dos serviços de saúde, dentre essas metas temos a prevenção do risco de queda, sendo a sexta meta de segurança do paciente (OLIVEIRA DU, et al., 2014; HOFFMEISTER LV e MOURA GMSS, 2015).

A mensuração do nível/grau de risco de queda do paciente pode influenciar nas escolhas de intervenções preventivas e devem ser feitas no momento da admissão do paciente com o emprego de uma escala adequada ao perfil de pacientes da instituição. Esta avaliação deve ser repetida diariamente até a alta do paciente (SANTOS MGS, et al., 2010). Tendo conhecimento desses dados, garantir assistência segura e de qualidade para os pacientes hospitalizados é uma das principais preocupações da enfermagem, que vem se apropriando de estratégias e práticas para a melhoria das suas ações de cuidado. (PEREIRA ES, 2018).

Diante da problemática, o interesse pela temática surgiu durante as aulas práticas desempenhadas no componente curricular "Enfermagem em Gestão e Gerenciamento" e "Enfermagem em Saúde do Idoso, que ocorreu em um hospital de referência em oncologia, localizado na cidade de Belém-PA. Nesse período foi observado e analisado que não era é realizado a avaliação do risco de queda em pacientes idosos por parte da equipe de saúde, ocorrendo apenas à notificação do evento, conforme se observou o que não corrobora com os pressupostos da Portaria no 2.095, de 24 de setembro de 2013 (BRASIL, 2013).

Assim, esse estudo possuiu como objetivo sensibilizar os profissionais de saúde de uma clínica neurológica quanto à importância da avaliação do risco de queda, no ambiente hospitalar, por intermédio de uma ação de educação permanente.

\section{RELATO DA EXPERIÊNCIA}

O estudo foi realizado em um hospital público de referência na cidade de Belém-PA. A pesquisa se deu a partir da observação sistemática da realidade em uma clínica de neurologia, feita por cinco acadêmicos do curso de graduação em enfermagem de uma universidade pública do estado do Pará, durante as aulas práticas do componente curricular "Gestão e gerenciamento dos serviços de saúde e enfermagem hospitalar", que ocorreu no período de 26 de fevereiro a 14 de março de 2019, sob supervisão de uma docente.

Fundamentado no Arco de Maguerez, na primeira etapa de observação sistemática da realidade, constatou-se que a referida clínica não possuía método de avaliação dos pacientes idosos quanto ao risco de queda, visto que esses pacientes se encontram mais vulneráveis a este risco. $\mathrm{Na}$ etapa de teorização, foi realizada busca no acervo bibliográfico para levantamento teórico referente ao risco de queda em idosos. Como hipótese de solução, foi organizada uma ação de educação permanente, referente ao risco de queda em idosos, tendo como público alvo a equipe de enfermagem da referida clínica de neurologia. 
A realização da quinta etapa do Arco de Maguerez, o retorno à realidade, estava prevista para o dia 14 de março de 2019, pelo turno da tarde, na sala da equipe com a equipe de enfermagem, porém, neste dia ocorreram imprevistos no hospital que acarretaram na sobrecarga de tarefas e consequentemente houve a indisponibilidade da enfermeira e do restante da equipe, que não compareceram ao momento de socialização, impossibilitando a realização da ação de educação permanente acerca da prevenção do risco de queda, meta 6 de Segurança do Paciente que dispõe sobre a avaliação individualmente de todos os pacientes e identificar aqueles que apresentam uma propensão maior a sofrerem quedas (SOARES EA, et al., 2019) como os pacientes idosos na Clínica Neurológica.

Os produtos advindos do planejamento da ação foram um folder, produzido no programa Microsoft Publisher 2013, e um slide, produzido no programa Microsoft PowerPoint 2013, que continham perguntas e afirmações com respostas verdadeiras ou falsas, para interagir com a equipe e mensurar a eficácia da ação que seria realizada com a equipe de enfermagem.

A frente do folder continha o tema da ação, informações sobre os autores do material e da instituição promotora. Além disso, também continha alguns cuidados que poderiam ser realizados pela equipe a fim de reduzir o risco e ocorrência de quedas na clínica, visto que não tinham nenhum método ou instrumento de avaliação e identificação desse risco, apenas notificavam quando a queda acontecia (Figura 1).

Figura 1 - Frente do folder.

\section{CUIDADOS PARA PREVENIR O RISCO DE QUEDA}

\footnotetext{
Para prevenir episódios de queda no ambiente hospitalar, é fundamental a proatividade da equipe multidisciplinar, além de educar e sensibilizar a familia do paciente. Outro ponto importante é manter 0 ambiente seguro, oferecendo infraestrutura adequada ao paciente.

Outras ações preventivas são:

- Avaliação do risco de quedas;

- Identificação do paciente em ris$\mathrm{co}$;

- Organização dos cuidados de higiene pessoal;

- Revisão da medicaçăo;

- Atenção aos calçados usados pelo paciente;

- Educaçāo familiar, paciente e profissionais.
}

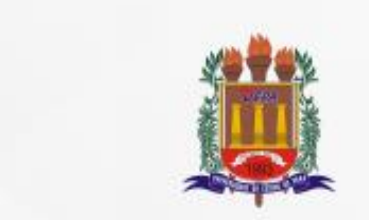

UNIVERSIDADE DO ESTADO DO PARA CENTRO DE CIENCIAS BIOLOGICAS E DA SAUUDE
CURSO DE GRADUACAO EM ENFERMAGEM CURSO DE GRADUAÇAO EM ENFERMAGEM
ATIVIDADES INTEGRADAS EM SAÚDE II Organizaçāo: CURSO DE GRADUAÇĀO EM ENFERMAGEM Subgrupo B1

Alicia Laura Lobo Modesto Beatriz Duarte de Oliveira Bruna Fonseca Rezende Larissa Renata Bittencourt Pantoja Ruhan da Conceiçāo Sacramento
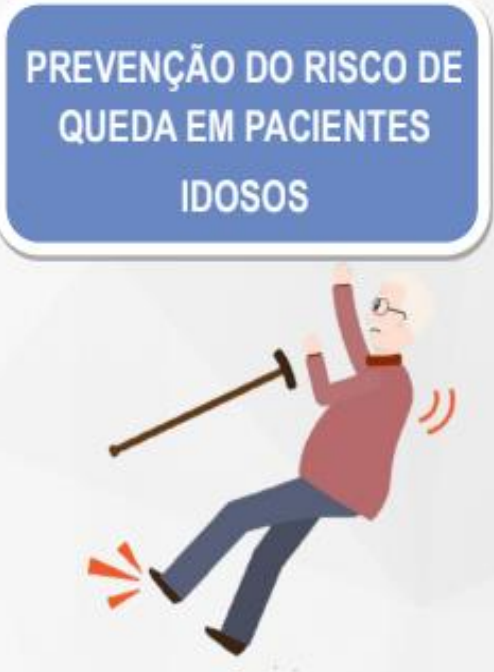

Fonte: Rezende BF, et al., 2019.

O verso do folder continha informações sobre o conceito de queda e os tipos de queda, sobre os fatores que contribuem para que a queda ocorra, muitos deles presentes na clínica, sobre os danos que poderiam ser ocasionados pela queda e, por fim, informações sobre a Escala de Queda de Morse, que não é uma escala utilizada na rotina do hospital, no entanto houve necessidade de inserir este tópico visto que alguns componentes da equipe de enfermagem não sabiam de sua existência e finalidade (Figura 2). 
Figura 2- Verso do folder.

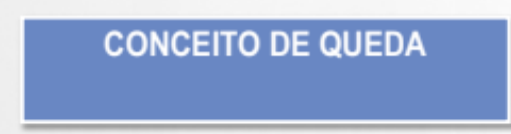

Considera-se queda quando 0 paciente é encontrado no chão ou quando, durante o deslocamento, necessita de amparo, ainda que não chegue ao chão. A queda pode ocorrer da própria altura, da cama ou de assentos tais como cadeira de rodas, higiênicas e vaso sanitário. As quedas são classificadas em:

Quedas acidentais: Ocorrem por fatores externos à pessoa, acontecendo a clientes sem risco de queda

Quedas fisiológicas não antecipáveis: Ocorrem em individuos sem fatores de risco para a queda, até que a primeira ocorra de fato, devido fatores fisiológicos como convulsões, perda de força, fraturas, patologias.

Quedas fisiológicas antecipáveis: Ocorrem em individuos com alterações fisiológicas e que apresentam risco de queda. Representam quase $80 \%$ do total de quedas e são as potencialmente preveniveis.

\section{FATORES CONTRIBUINTES PARA}

$$
\text { A QUEDA }
$$

- Estado Mental: confusão, desorientação, depressão, deficiências cognitivas;

- Mobilidade: astenia, desequilibrio, deficiências motoras;

- Medicação: medicamentos que atuam ao nivel do Sistema Nervoso Central;

- Necessidades fisiológicas (eliminação): doentes incontinentes e que necessitam de apoio;

- Idade: pacientes com idades $>60-65$ possuem maior risco de queda. 0 risco aumenta em doentes com idade superior a 80 anos.

\section{DANOS RELACIONADOS A QUE- DA}

- Danos graves: fraturas, Hematomas subdurais, sangramentos $e$ óbito;

- Impacto negativo (sequelas, medo e depressão);

- Aumento de permanência hospitalar;
- Aumento de custo da assistência;

- Redução da credibilidade na qualidade da assistência;

- Repercussão de ordem legal.

\section{ESCALA DE QUEDA DE MORSE}

\section{(EQM)}

A EQM é constituída por seis itens com duas ou três possibilidades de resposta para cada um, cada uma das respostas corresponde a um score que indicará o risco de queda.

Essa pontuação varia de 0 a 125 pontos, onde considera-se alto risco de queda quando o resultado obtido, através da aplicaçăo da escala, é igual ou superior a 45 pontos.

EQM deve ser efetuada no momento da admissão assim como quando existe alteraçăo da condiçăo clinica do cliente ou quando ocorre uma queda.

A aplicaçăo da EQM é uma atribuição do enfermeiro, no entanto toda a equipe multiprofissional é responsável por observar e sinalizar a presença de fatores que podem contribuir para que a queda ocorra.

Fonte: Rezende BF, et al., 2019.

Em um segundo momento, após a socialização com a equipe, foram utilizados slides a fim de realizar a mensuração da eficácia da ação, como dito anteriormente. O slide continha perguntas com afirmações assertivas de verdadeiras ou falsas elaboradas pelos próprios autores partir da leitura do Protocolo nacional de Segurança do Paciente e de outros autores que abordam a temática (BRASIL, 2013; GARCIA MB, 2016; SOARES EA, et al., 2019; GOMES MVDS, 2019; SANTOS MGS, et al., 2017; VACCARI E, et al., 2016).

É importante ressaltar que a escolha para abordagem de tal temática se deu diante a uma observação sistemática, por meio da qual foi detectada a ausência de instrumentos para a avaliação do risco de queda, como a escala de Morse, por exemplo. Por meio de relatos informais com membros da equipe de enfermagem da clínica, foi evidenciado que o evento adverso em questão era notificado para o núcleo de segurança do paciente após a ocorrência do mesmo. Não havendo cuidados e orientações para que medidas preventivas pudessem ser tomadas por parte da equipe e/ou acompanhantes dos pacientes.

\section{DISCUSSÃO}

Segundo Ilha S, et al. (2014) para a elaboração de um plano assistencial adequado, o profissional enfermeiro necessita identificar os fatores que são inerentes à ocorrência de quedas nos pacientes idosos. Dentre esses determinantes, destacam-se: os ambientais, cognitivos, fisiológicos, o uso de determinados medicamentos, bem como a idade. Tais fatos são corroborados por Oliveira RM, et al. (2017), o qual evidencia que é de extrema importância que profissionais enfermeiros tenham conhecimento dos fatores predisponentes que elevam o risco para a ocorrência de quedas em pacientes da $3^{\text {a }}$ idade, para a realização de uma assistência de qualidade pautada na segurança do paciente.

Assim, como resultados esperados os acadêmicos objetivavam, por meio da realização da ação, a sensibilização da equipe quanto a importância da avaliação do risco de queda para os pacientes idosos, bem como para a prestação de cuidados que pudessem evitar tal evento, uma vez que tratava-se de um setor de clinica neurológica, onde múltiplos fatores elevavam a pré-disposição de ocorrência do evento adverso, como 
por exemplo os déficits cognitivos, os quais, segundo Oliveira RM, et al. (2017), podem ocasionar certa dificuldade por parte dos idosos em avaliar a real situação em que se encontram, levando-os a uma percepção equivocada de suas capacidades e predispondo-os à ocorrência da queda.

É evidente que o profissional enfermeiro desempenha papel fundamental para a prevenção do risco de quedas e, para isso, é necessário que se faça aplicabilidade de conhecimentos específicos para intervir sobre a realidade objetivando a redução da ocorrência do evento adverso durante o período de internação do paciente idoso. Assim, o uso de estratégias, como ações de educação permanente, ganha notoriedade para a capacitação da equipe de enfermagem frente aos protocolos de avalição, com vistas a possibilitar a Segurança do Paciente (SP) da $3^{\text {a }}$ idade (ILHA S, et al., 2014).

Nogueira A, et al. (2012) afirma que, além da equipe de Enfermagem, é importante que outros profissionais de saúde, como os médicos, sejam capacitados e para a identificação dos fatores de risco para queda. Para isso, destaca-se a importância da utilização dos protocolos criados pelo Ministério da Saúde, a fim de impulsionar a SP em senis, bem como de outros públicos (BRASIL, 2013b).

O protocolo para a prevenção do risco de queda visa a redução da ocorrência de tal eventualidade em pacientes hospitalizados, bem como os danos provenientes deste evento adverso, por meio da execução de medidas que possibilitem a análise do risco apresentado por cada paciente para a prestação do cuidado multiprofissional pautado nos preceitos das metas de segurança do paciente, de maneira a objetivar um ambiente seguro, além de promover a importante educação do paciente, familiares e profissionais com vistas a preservar a dignidade do paciente (BRASIL, 2013a). No entanto, a não efetivação de tais práticas, colocam em xeque a credibilidade dos serviços de saúde no que tange ao risco de queda e SP dos pacientes em suas diversas esferas e, principalmente, para os idosos por serem vulneráveis.

\section{CONSIDERAÇÕES FINAIS}

O presente estudo apresenta limitações, uma vez que os objetivos iniciais não foram alcançados por questões advindas do cotidiano da equipe do local onde realizaram-se as atividades práticas do componente curricular de Gestão e Gerenciamento, as quais não estavam sob governabilidade dos acadêmicos e da docente supervisora. Contudo, por parte dos acadêmicos, considera-se a pesquisa como satisfatória no que tange ao conhecimento adquirido referente aos fatores predisponentes para o risco de queda em pacientes idosos hospitalizados, bem como as atribuições do enfermeiro na intervenção para a redução do evento possibilitando a prestação de uma assistência de qualidade e segura aos pacientes senis.

\section{REFERENCIAS}

1. BERBEL NAN. As metodologias ativas e a promoção da autonomia de estudantes. Semina: Ciências Sociais e Humanas, 2011; 32(1): 25-40.

2. BRASIL. Ministério da Saúde. Anvisa. Protocolo Prevenção de Queda. Brasília: Ministério da Saúde, $2013 a$.

3. BRASIL. Ministério da Saúde. Gabinete do Ministro. Portaria no 2.095, de 24 de setembro de 2013. Aprova os Protocolos Básicos de Segurança do Paciente. Diário Oficial da União, Brasília, DF, 25 set. 2013b. Seção 1, p. 113.

4. GARCIA MB. Prevenindo quedas. Entendendo a escala de morse. Revista das Semanas Acadêmicas, 2016; 3(3).

5. GOMES MVDS. Conhecimento de graduandos de Enfermagem sobre as seis metas internacionais de segurança do paciente. Monografia (Trabalho de Conclusão de Curso-) - Curso de Graduação em Enfermagem, Faculdade de Ciências da Educação e Saúde, Centro Universitário de Brasília, Brasília, 2019; 18 p.

6. HOFFMEISTER LV, MOURA GMSS. Uso de pulseiras de identificação em pacientes internados em um hospital universitário. Revista Latino-americana de Enfermagem, jan.-fev. 2015; 23(1): 36-43.

7. ILHA S et al. Quedas em idosos: Reflexão para enfermeiros e demais profissionais. Revista de Enfermagem [UFPE online], 2014; 8(6): 1791-1798.

8. NOGUEIRA A et al. Risco de queda nos idosos: educação em saúde para melhoria da qualidade de vida. Revista Práxis, ago. 2012; 4(8): 77-82.

9. OLIVEIRA DU et al. Avaliação de quedas em idosos hospitalizados. Revista de Enfermagem [UFPE online], nov. 2017; 11(11): 4589-4597.

10. OLIVEIRA RM et al. Estratégias para promover segurança do paciente: da identificação dos riscos às práticas baseadas em evidências. Escola Anna Nery Revista de Enfermagem, jan.-mar. 2014; 18(1): 122-129.

11. PEREIRA ES. INTERVENÇÕES DE ENFERMAGEM EM IDOSOS HOSPITALIZADOS COM RISCO DE QUEDA: Um estudo de mapeamento. Dissertação (Mestrado em Ciências do Cuidado em Saúde) - Escola de Enfermagem Aurora de Afonso Costa, Universidade Federal Fluminense, Niterói, 2018; 132 p. 
12. SANTOS DS et al. Segurança do paciente: implantação de pulseiras de identificação na UTI adulto. Monografia (Trabalho de Conclusão de Curso Especialização) - Universidade Federal de Santa Catarina. Centro de Ciências da Saúde. Programa de Pós-graduação em Enfermagem. Linhas de Cuidado em Urgência e Emergência, Florianópolis, 2017; $32 \mathrm{p}$.

13. SANTOS MGS et al. Protocolos de Enfermagem: identificação de risco de prevenção de quedas. Rio de Janeiro: Hemorio, 2010; $17 \mathrm{p}$.

14. SOARES EA et al. Cultura de Segurança do Paciente e a Prática de Notificação de Eventos Adversos. Revista Eletrônica Acervo Saúde, 2019; (36): 1657-1657

15. VACCARI E et al. Segurança do paciente idoso e o evento queda no ambiente hospitalar. Cogitare Enfermagem, 2016; $21(5)$.

16. VICTOR MAG et al. Quedas em pacientes cirúrgicos: subsídios para o cuidado de enfermagem seguro. Revista de Enfermagem [UFPE online], out. 2017; 11(10): 4027-4035 